\title{
Nephrologische Weiterbildung in Ulm Das Erfolgsmodell wird fortgeführt
}

Die Fort- und Weiterbildung für nephrologisches Personal hat in Ulm eine lange und erfolgreiche Tradition. Seit 1980 wird hier die berufsbegleitende Fachweiterbildung zur Erlangung der Bezeichnung „Fachschwester/Fachpfleger für Nephrologie“ durchgeführt. Seit 1995 ist die Weiterbildungsstätte Ulm vom Regierungspräsidium Tübingen staatlich anerkannt. Die 2-jährige berufsbegleitende Fachweiterbildung basiert auf der Verordnung des Sozialministeriums zur Weiterbildung in den Berufen der Krankenpflege und Kinderkrankenpflege auf dem Gebiet der Nephrologie, der Empfehlung der Deutschen Krankenhausgesellschaft vom März 1995 und ist an den Kernlehrplan der „European Dialysis and Transplant Nurses Association/European Renal Care Association“ (EDTNA/ERCA) angelehnt.

Die Leitung der Weiterbildungsstätte besteht aus einer gemeinsamen ärztlichen und pflegerisch-pädagogischen Leitung. Momentan stehen uns 115 pflegerische und ärztliche Lehrkräfte für den theoretischen Unterricht sowie 6 ausgebildete Mentoren, Unterrichtspfleger, leitende Pflegekräfte und weitere Mitarbeiter aus dem multidisziplinären Team (Techniker, Ökotrophologen etc.) für den praktischen Unterricht zur Verfügung. 720 Stunden theoretischen und praktischen Unterricht müssen die Teilnehmer in den 2 Jahren absolvieren. Alle Referenten erhalten vor dem Unterricht einen Lernzielkatalog und liefern ein schriftliches Manuskript für die Teilnehmer ab.

\section{Klar strukturierter Unterricht ...}

Die Weiterbildung erfolgt in 8 Blöcken zu je 5 Unterrichtstagen. Es werden 40 Unterrichtstage zentral in Ulm abgehalten und 3 Tage für die 3-geteilte schriftliche, mündliche und praktische Prüfung reserviert.

Im Unterricht werden theoretische und praktische Unterrichtsinhalte eng miteinander verknüpft. Die Themenkomplexe werden als abgeschlossene Mo- dule unterrichtet. Dies sind Transplantationsmedizin, Heimdialyse, Ernährungsmedizin, Apheresetherapie usw. Ergänzend zu den Unterrichtseinheiten bekommen die Weiterbildungsteilnehmer nach jedem Block Aufgabenstellungen zur Bearbeitung, die helfen sollen, das Gelernte bis zum nächsten Block umzusetzen und anzuwenden. Die Verknüpfung von theoretischen Lerninhalten, wie die Pflegeplanung oder Einführung von Standards, wird so direkt in die Praxis im eigenen Zentrum transferiert. Der fachpraktische Unterricht erfolgt anhand des Praxisbegleitheftes in der entsendenden Einrichtung. Hospitationen erfolgen nach Absprache.

\section{... deckt ein breites Aufgaben- gebiet ab}

Die Weiterbildung soll Gesundheitsund Krankenpflegekräfte mit ihren vielfältigen Aufgaben in den verschiedenen Gebieten der akuten und chronischen nephrologischen Pflege vertraut machen und ihnen die zur Erfüllung dieser Aufgaben erforderlichen speziellen Kenntnisse, Fertigkeiten, Verhaltensweisen und Einstellungen vermitteln. Gerade die umfassende Ausbildung in

\section{Fachweiterbildung}

\section{Teilnahmevoraussetzungen}

1. abgeschlossene 3-jährige Ausbildung zum Gesundheits-/ Krankenpfleger

a. Zeugnis der Gesundheits- bzw. Krankenpflegeausbildung

b. Erlaubnis zur Führung der Berufsbezeichnung nach dem Krankenpflegegesetz

2. mindestens 2 Jahre Berufserfahrung, davon mindestens 6 Monate in der Nephrologie

Kosten für die Fachweiterbildung

Beginn des nächsten Weiterbildungskurses
4800 Euro

Februar 2009 


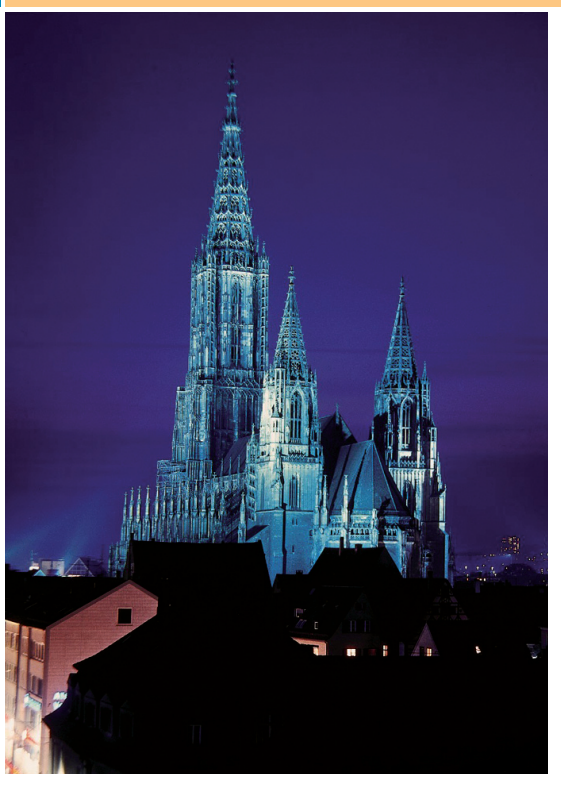

den verschiedenen nephrologischen Teilgebieten ermöglicht den Teilnehmern, Zusammenhänge besser zu verstehen und auch an andere (Mitarbeiter und Patienten) weiterzugeben.

Da die Teilnehmer aus verschiedenen Einrichtungen kommen (nephrologische und Transplantationsstation, akute und chronische Dialyseeinrichtung, Kliniken und privaten Praxen), gewinnt die Ausbildung durch den Erfahrungsaustausch und die gegenseitige Hospitation in den vielen kooperierenden Einrichtungen sowie die speziellen Unterrichtsangebote (Kinästhetik, geriatrischer Unterricht in einem geriatrischen Zentrum, Besuche von Produktionsstätten für Dialysebedarf etc.) an Substanz. Die Anforderung an die Qualifikation des Pflegepersonals in Dialyseeinheiten wird im Dialysestandard 2006 beschrieben. Die Teilnehmerzahl ist auf 15 Personen begrenzt.

\section{Seminare}

Weiterhin finden regelmäßig 2-5-tägige Seminare statt. Alle Veranstaltungen werden in kleinem Kreis (maximal 30 Teilnehmer) durchgeführt, um Diskus- sionen Raum zu geben. Die umfangreichen Seminarunterlagen werden den Teilnehmern zu Beginn der Schulungen ausgehändigt.

\section{Herbstkurse für das multidisziplinäre Team}

Seit 1996 führen wir jährlich unsere „Herbstkurse für das multidisziplinäre Team“ („Nephrologie 1“ vom 10.14.11.2008 und „Nephrologie 2“ vom 17.-21.11.2008) von jeweils einer Woche Dauer recht erfolgreich durch. Zielgruppe sind alle Mitarbeiter, die eine 2-jährige Fachweiterbildung nicht absolvieren können oder wollen, Mitarbeiter nach der Einarbeitung, die ihr Wissen vertiefen und erweitern möchten, oder langjährige Mitarbeiter, die ihr Wissen auf einen aktuellen Stand bringen wollen. Insbesondere sind hiermit nephrologisch tätige Arzthelferinnen, Pflegepersonal von nephrologischen oder Transplantationsstationen, aber auch Assistenzärzte gemeint.

Der Unterricht ist sehr praxisnah gegliedert, und es erfolgen nach den theoretischen Teilen auch praktische Übungen vor Ort. Jeder Kurs schließt mit einer Prüfung ab, die anschließend als Wiederholung der Lerninhalte besprochen wird. Bestehende Lücken können anhand der umfangreichen schriftlichen Schulungsunterlagen nachgearbeitet werden. Die Kosten betragen 610 Euro pro Woche, inklusive Verpflegung und Seminarunterlagen.

\section{Arzthelfer(in) in der Dialyse}

Die Weiterbildungsstätte Ulm wurde vor sechs Jahren von der Landesärztekammer Baden-Württemberg zur Durchführung des Fortbildungscurriculums „Arzthelfer(in) in der Dialyse“ und zur Abnahme der Prüfung ermächtigt. Seit 2002 führen wir diese Weiterbildung jährlich als 2-wöchigen Blockun-

\section{Komplettes Programm zum 30. AfnP-Symposium in dieser Ausgabe}

Auch in diesem Jahr finden Sie das Programm zum 30. AfnP-Symposium am 25. und 26. Oktober 2008 in Fulda als Beilage in dieser Ausgabe der Dialyse aktuell. Ärzte erhalten für die Teilnahme der gesamten Veranstaltung 15 Fortbildungspunkte der Kategorie B. Pflegekräfte können Fortbildungspunkte der freiwilligen Registrierungsstelle gutgeschrieben bekommen. Alle Teilnehmer die keine Registrierungspunkte sammeln erhalten eine Teilnahmebescheinigung (ohne Registrierungspunkte) für die 2 Tage mit Ihrer Eintrittskarte an der Kasse. Hoffentlich spricht auch in diesem Jahr das vielseitige Programm alle im nephrologischen Bereich tätigen Berufsgruppen an. Das umfassende Programm findet in 7 Räumen parallel statt.

terricht mit auf 100 Stunden erweitertem Stundenumfang durch (Pflicht sind laut Curriculum 80 Stunden). Aufgrund unserer langjährigen Fortbildungserfahrung haben wir den Stundenumfang erweitert und mit Themen ergänzt, die uns wichtig erscheinen. Dies sind beispielweise Ernährung, Diabetes, geriatrische Nephrologie und kardiale Komplikationen. Das Praktikum erfolgt anhand des Praktikumbegleitheftes. Die Abschlussprüfung findet Ende Januar des folgenden Jahres statt. In der Zielgruppe befinden sich Arzthelfer, Gesundheits- und Krankenpflegekräfte mit mindestens 6 Monaten Berufserfahrung in der Dialyse. Der nächste Kurs startet am 10.11.2008.

Marion Bundschu, Leitung der Weiterbildungsstätte Ulm

\section{Literatur}

1 Deutsche Arbeitsgemeinschaft für Klinische Nephrologie e. V. in Zusammenarbeit mit dem Verband Deutscher Nierenzentren der DdnÄ e. V. und der Arbeitsgemeinschaft für Pädiatrische Nephrologie (APN). Dialysestandard 2006

2 DKG-Empfehlung zur Weiterbildung für Krankenpflegepersonal in der Nephrologie. Das Krankenhaus 1995; 5

3 European Standards for Nephrology Nursing Practice, Gent, 1994

4 Küntzle A, Hrsg. European Dialysis und Transplant Nurses Association/European Renal Care Association (EDTNA/ERCA), Kernlehrplan, 1994

5 Verordnung des Sozialministeriums über die Weiterbildung in den Berufen der Krankenpflege/Kinderkrankenpflege auf dem Gebiet der Nephrologie. Weiterbildungsverordnung Nephrologie vom 19.12.2000, Gesetzblatt für Baden Württemberg

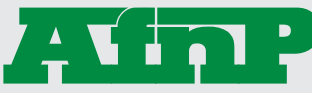

Arbeitsgemeinschaft für nephrologisches Personal e. V.

So können Sie uns erreichen:

AfnP Geschäftsstelle

Käppelesweg 8

89129 Langenau

Tel.: $07345 / 22933$

Fax: $07345 / 7540$

E-Mail: info@afnp.de

Internet: www.afnp.de

Vorstand der AfnP e.V.

- Marion Bundschu (1. Vorsitzende)

- Hans-Martin Schröder (stellv. Vorsitzender)

- Brigitte Franke-Paar (Schatzmeisterin)

- Helga Damaschke (Schriftführerin) 